\title{
Preparation and Properties of Magnetic Hematite Core/Alumina Shell Nanospheres
}

\author{
Qing-Tao FU ${ }^{a^{*}}$, Ting-Ting $\mathrm{HE}^{\mathrm{b}}$, Rong-Jian YING \\ College of Chenistry and Chemical Technology, LinYi University , LinYi 276000, China \\ afuqingtao@lyu.edu.cn, bhetingheyi@163.com, ‘fqt888@126.com \\ * Corresponding author
}

Keywords: $\alpha-\mathrm{Fe}_{2} \mathrm{O}_{3}$, Alumina Nanosphere, Core-shell, Stearic Acid

\begin{abstract}
Magnetic core/alumina shell (MFeCA) structured nanospheres were obtained by a hematite core/alumina precursor shell (HFeCAP) nanosphere precursor. A uniform alumina layer was coated on the surface of $\alpha-\mathrm{Fe}_{2} \mathrm{O}_{3}$ by the self-assembly of a stearic acid/alumina isopropoxide complex. Hematite cores of the nanospheres were then reduced under $\mathrm{H}_{2} / \mathrm{N}_{2}$ gas flow to produce the MFeCA nanospheres. Transmission electron microscopy images showed that the products possessed a distinct core-shell structure. $\mathrm{N}_{2}$ sorption measurements showed that the uniform accessible pore size was $4.0 \mathrm{~nm}$. The surface area was $140 \mathrm{~m}^{2} / \mathrm{g}$ and pore volume was $0.22 \mathrm{~cm}^{3} / \mathrm{g}$. Saturation magnetization values indicated the product had a high degree of magnetization (50.2 emu/g).
\end{abstract}

\section{Introduction}

Core-shell nanosphere research has recently received extensive attention, as the functionality of these structures allows their use in various applications [1-7]. The fabrication of microspheres from alumina and magnetic particles has been reported, and is of interest due to their unique magnetic responsivity and excellent physical strength [8-10]. Feng et al. [8,9] previously showed that single phase $\mathrm{NiFe}_{2} \mathrm{O}_{4}$ ferrite spinel core/porous alumina shell microspheres could be used in a magnetically stabilized bed MSB as a catalyst or catalyst support. $\mathrm{Ni}_{0.8} \mathrm{Co}_{0.2} \mathrm{Fe}$ oxide has been used as a core for magnetic alumina microspheres, prepared by the hydrolysis of aluminum isopropoxide. Li et al. [10] developed $\mathrm{Fe}_{3} \mathrm{O}_{4} @ \mathrm{Al}_{2} \mathrm{O}_{3}$ magnetic core-shell nanospheres, which were applied to enrich phosphopeptides from the tryptic digest of phosphoproteins (casein and ovalbumin).

Such studies generally yielded larger particles with diameters from hundreds of nanometers to micrometers. Hematite particles can be prepared in a wide range of sizes and shapes, by aging ferric salt solutions [11]. The surface of hematite particles can be coated directly and uniformly with alumina due to its abundant hydroxyl groups. Prepared $\alpha-\mathrm{Fe}_{2} \mathrm{O}_{3}$ particles exhibit single-crystal structures with relatively uniform sizes in the range 180-360 $\mathrm{nm}$. In this study, we fabricated uniform nanocomposite spheres with a hematite core/alumina precursor shell (HFeCAP) structure. The alumina shell was formed via a combination of the aluminum isopropoxide sol-gel process and stearic acid self-assembly. The nanospheres hematite core was reduced in gas flow mixture of $\mathrm{H}_{2}$ and $\mathrm{N}_{2}$, to produce the MFeCA (magnetic core/alumina shell) nanosphere and remove the organic group.

\section{Materials and Methods}

\subsection{Materials}

Ferric nitrate $\left(\mathrm{Fe}\left(\mathrm{NO}_{3}\right)_{3} \cdot 9 \mathrm{H}_{2} \mathrm{O}\right)$, polyvinylpyrrolidone (PVP), aqueous ammonia (28 wt\%), 1-propanol, aluminum isopropoxide $\left(\mathrm{Al}\left[\mathrm{OCH}\left(\mathrm{CH}_{3}\right)_{2}\right]_{3}\right)$, and ethanol were all of analytical grade and were purchased from the Shanghai Chemical Corp.

Hematite particles were hydrothermally synthesized by a surfactant-directing approach 
previously reported [11]. In a typical synthesis of hematite core/porous alumina shell composite particles, $0.1 \mathrm{~g}$ of hematite particles (diameter 100-200 nm) were dispersed by ultrasonication into $100 \mathrm{~mL}$ of 1-propanol containing stearic acid. $0.2 \mathrm{~g}$ of aluminum is opropoxide was added under vigorous stirring, and the reaction was stirred at $70^{\circ} \mathrm{C}$ for $6 \mathrm{~h}$. The resulting stearic acid-incorporated core-shell particles were separated by centrifugation, washed with ethanol and dried at room temperature. This intermediate product was named HFeCAP (hematite core/alumina precursor shell spheres). The thermal treatment of HFeCAP in air at $673 \mathrm{~K}$ for $3 \mathrm{~h}$ and subsequently in a mixed $\mathrm{H}_{2}(4 \mathrm{vol} \%) / \mathrm{N}_{2}$ atmosphere at $673 \mathrm{~K}$ for $3 \mathrm{~h}$, yielded the final magnetic core/alumina particles (MFeCA).

\subsection{Analysis Methods}

X-ray powder diffraction (XRD) was carried out using a PANalytical $X^{\prime}$ Pert Pro MPD diffractometer. The magnetic properties of the samples were measured at room temperature using a Lake Shore vibrating sample magnetometer (VSM). Particle morphology and size were examined using a Hitachi S-3400 scanning electron microscope (SEM) and a JEM-2100UHR transmission electron microscope (TEM). Low temperature $\mathrm{N}_{2}$ sorption experiments were carried out using a Micromeritics ASAP-2020 Quantachrome Autosorb-1 system.

\section{Results and Discussion}

Stearic acid was used as an anion template to grow a stearic acid/alumina precursor layer on the $\alpha-\mathrm{Fe}_{2} \mathrm{O}_{3}$ polyhedron surface, thus forming a core-shell structure. After calcination to remove the stearic acid template, porous alumina shells were formed. A TEM image showed a typical distinct core/shell structure $\left(\alpha-\mathrm{Fe}_{2} \mathrm{O}_{3}\right.$ core/alumina precursor shell) (Fig.1(a)). The thickness of the porous alumina shell was $30 \mathrm{~nm}$. The energy-dispersive X-ray (EDX) spectrum of the $\mathrm{Fe}_{2} \mathrm{O}_{3} @ \mathrm{Al}_{2} \mathrm{O}_{3}$ precursor core-shell microspheres (Figure 1(b)) revealed the existence of Fe, $\mathrm{Al}, \mathrm{O}$ and $\mathrm{C}$, further indicating an alumina precursor shell had been formed on the $\mathrm{Fe}_{2} \mathrm{O}_{3}$ microsphere surface. Elemental analysis indicated an $\mathrm{Al} / \mathrm{Fe}$ mass fraction of 0.36 , which was similar to that of the synthesis mixture.
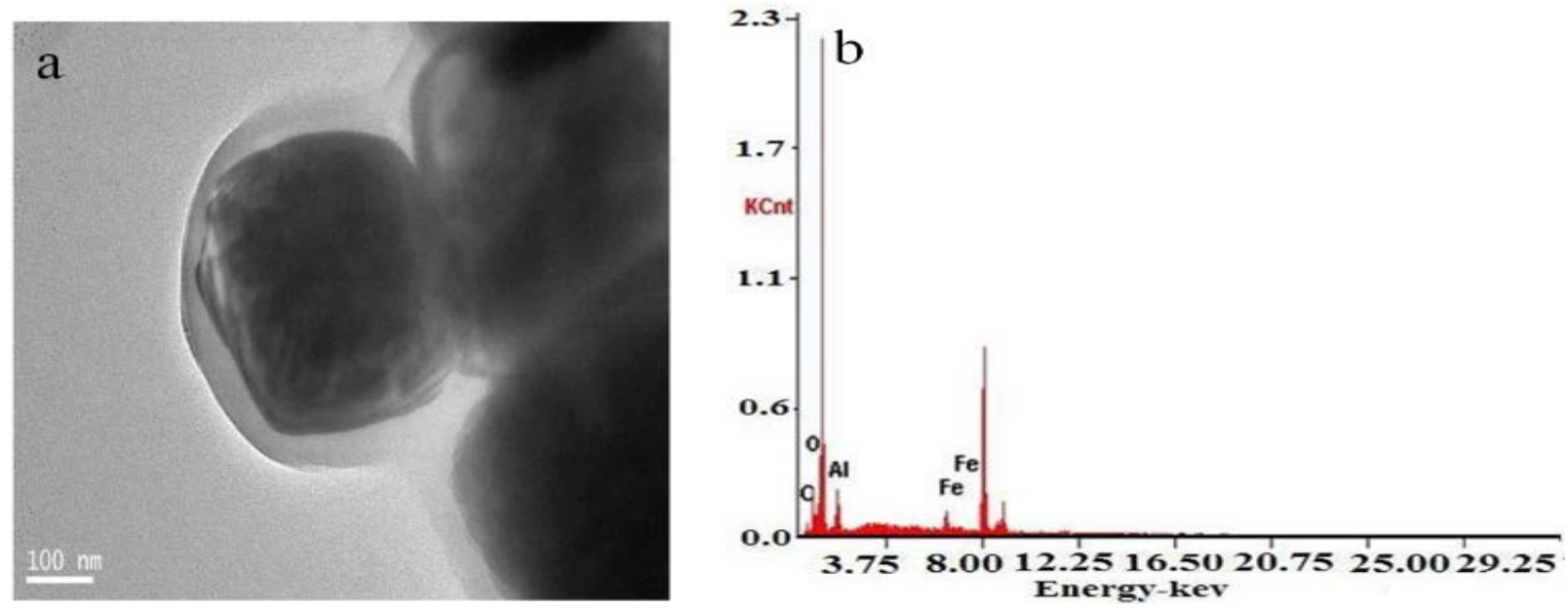

Fig.1 (a) TEM image of HFeCAP nanospheres; (b) EDX of HFeCAP nanospheres 


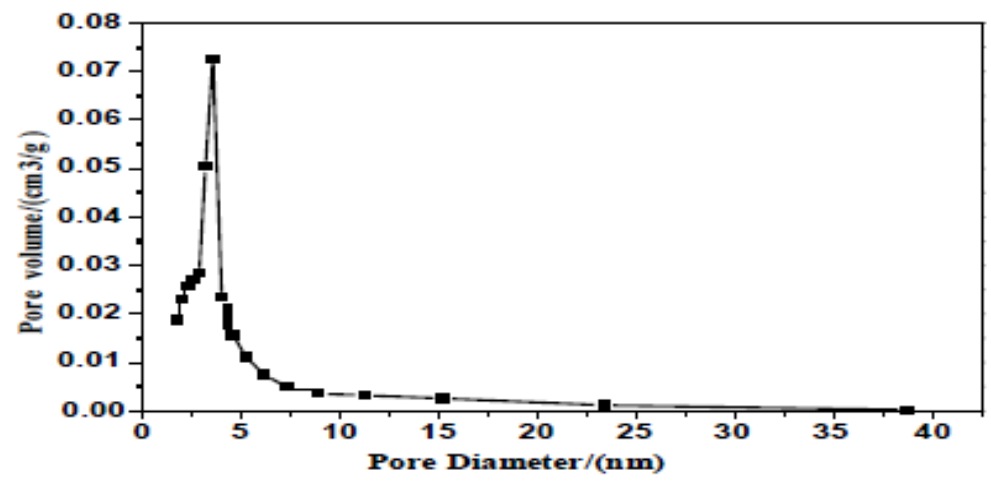

Fig.2 $\mathrm{N}_{2}$ adsorption isotherms of MFeCA nanospheres

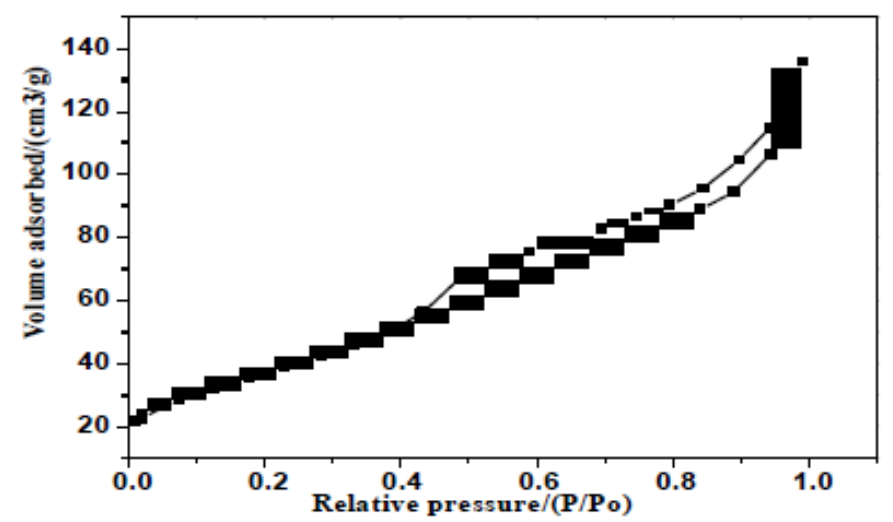

Fig.3 Pore size distributions for MFeCA nanosphere

$\mathrm{N}_{2}$ adsorption-desorption isotherms and the pore size distribution of the sample are shown in Fig.2 and 3, respectively. The sample presented type II isotherm behavior with an obvious hysteresis loop [12]. The appearance of the superposition of type H1 and H3 hysteresis loops in the isotherm indicated the presence of both tubular and parallel slit-shaped capillary pores. These were caused by escaping gas during calcination, and the stacking of alumina nanocrystallites. Physisorption measurements revealed a high BET surface area $\left(140 \mathrm{~m}^{2} / \mathrm{g}\right)$ and high pore volume $\left(0.22 \mathrm{~cm}^{3} / \mathrm{g}\right)$. A narrow pore size distribution centered at $4.0 \mathrm{~nm}$ pore diameter indicated a small number of micropores and a larger number of mesopores.

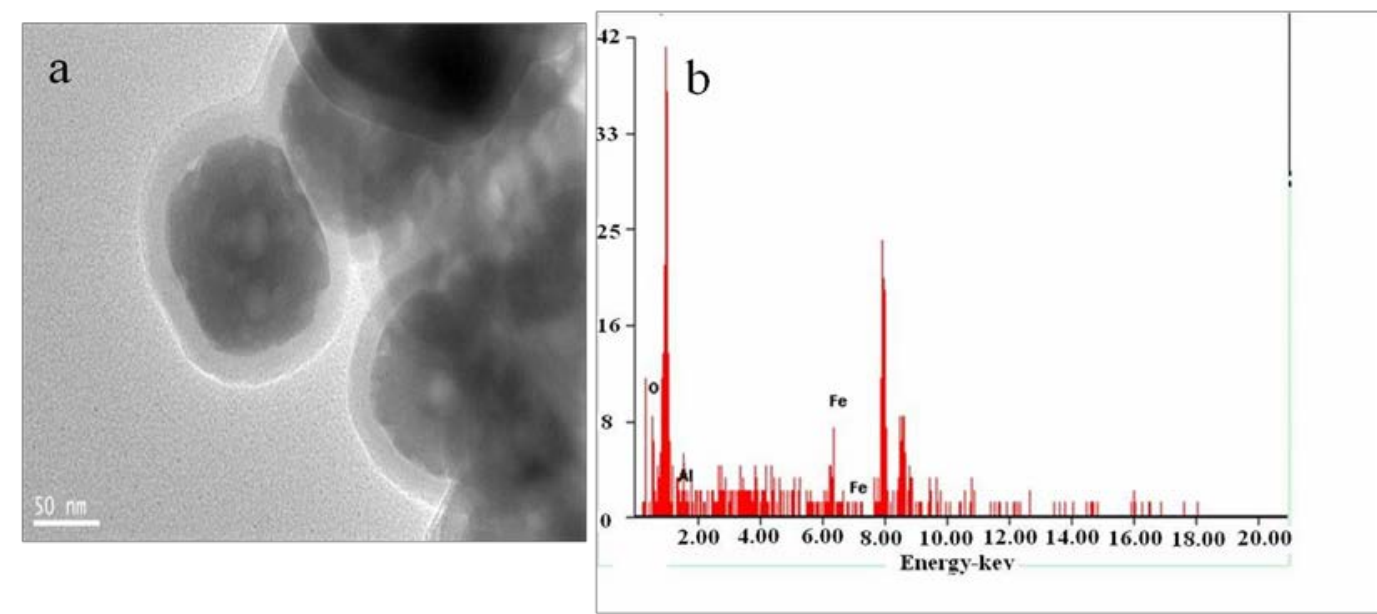

Fig.4 (a) TEM image of MFeCA nanospheres; (b) EDX of MFeCA nanospheres

After reduction, the color of the sample powder changed from red to deep black, consistent with the reduction of the hematite cores. A TEM image of the MFeCA nanospheres 
(Fig. 4(a)) showed that most cores shrunk while their shell remained unbroken. The EDX spectrum of the $\mathrm{Fe}_{2} \mathrm{O}_{3} @ \mathrm{Al}_{2} \mathrm{O}_{3}$ core-shell nanospheres (Fig.4(b)) revealed the existence of Fe, $\mathrm{Al}$, and $\mathrm{O}$, further confirming the formation of the alumina shell on the $\mathrm{Fe}_{3} \mathrm{O}_{4}$ microsphere surface. Elemental analysis indicated an $\mathrm{Al} / \mathrm{Fe}$ mass fraction of 0.34 , which was consistent with that of the synthesis mixture.

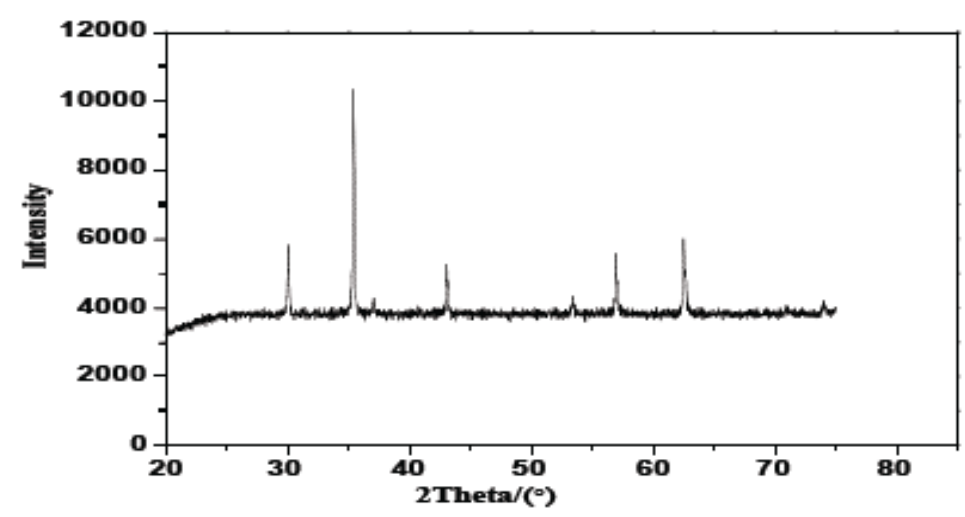

Fig.5 XRD pattern of MFeCA nanospheres

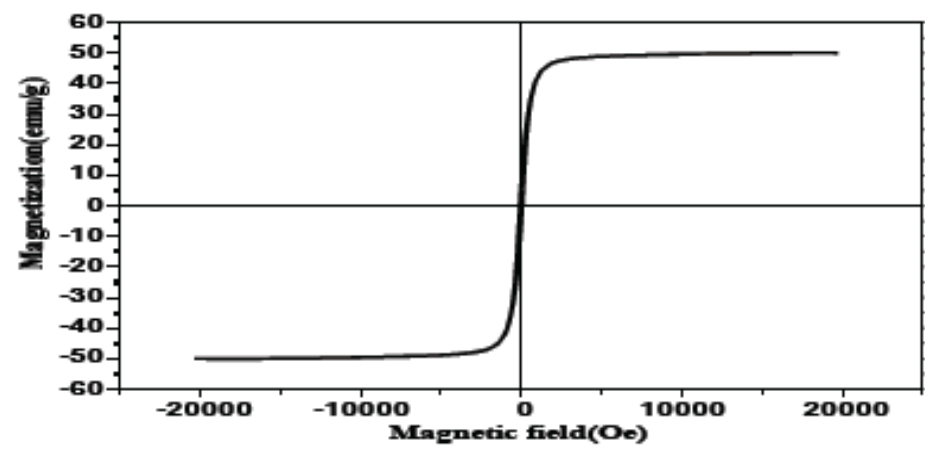

Fig.6 Magnetization hysteresis loops of MFeCA nanospheres

The composition of the reduction product could be controlled by altering the reduction temperature or time, as described previously [13]. After reduction, close agreement between dominant XRD peaks and standard PDF data confirmed the nanosphere cores were reduced iron particles (Fig.5). No alumina diffractions were observed, in agreement with the literature [14], which indicated an amorphous structure. The room-temperature magnetization curve (Fig.6) showed paramagnetism, which depicted a strong magnetic response to the varying magnetic field. The intrinsic ferromagnetism of MFeCA provided a straightforward way to separate particles from miscible liquids compared with $\mathrm{Fe}_{3} \mathrm{O}_{4} @ \mathrm{Al}_{2} \mathrm{O}_{3}$ spheres. The saturation magnetization value of the MFeCA nanospheres ( $50.2 \mathrm{emu} / \mathrm{g})$ could be adjusted by controlling the reduction temperature or time, according to the typical reduction processes of hematite.

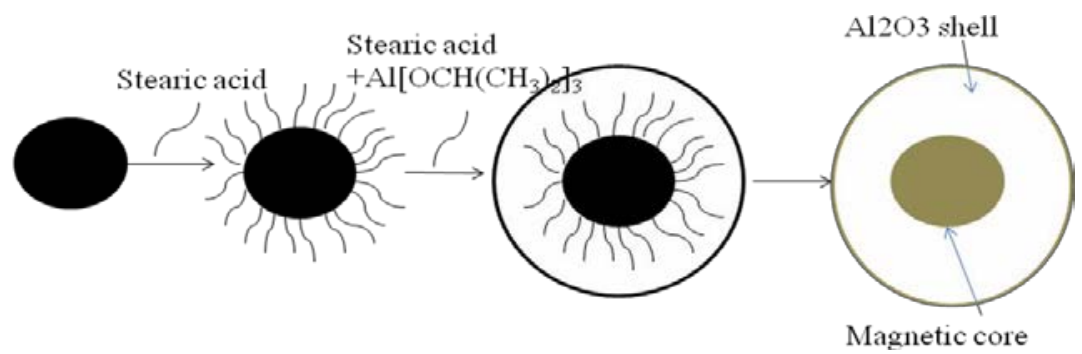

Fig.7 The formation process of the MFeCA nanospheres 
Fig.7 illustrates the formation process of the $\mathrm{Fe}_{2} \mathrm{O}_{3} @ \mathrm{Al}_{2} \mathrm{O}_{3}$ core-shell nanosphere structure. We propose that HFeCA nanospheres were formed by the self-assembly of composite $\alpha-\mathrm{Fe}_{2} \mathrm{O}_{3}$-surfactant particles and surfactant/alumina complexes. The nanosphere/stearic acid capped particles contained a negative charge [13], due to the carboxyl groups of the anionic surfactant. Hydrolyzed alumina and surfactant templates produce complexes, and their surfaces contain aluminum carboxyl groups. The nanosphere-surfactant particles acted as a core to attract alumina/surfactant complexes through electrostatic interactions, followed by surfactant directed self-assembly of alumina/surfactant phases. A detailed explanation is as follows: hydrolyzed aluminum isopropoxide monomers condense to form oligomeric alumina precursors. When the precursors reach a certain size, precursors and surfactants are assembled to produce an alumina shell on the surface of the nanosphere-surfactant composites. The residual alumina/surfactant complexes then react preferentially with alumina hydroxyl groups on the surface of the particles that have been generated, preventing the formation of any extra particles, thus forming uniform magnetic core-shell alumina nanospheres.

\section{Conclusions}

Uniform magnetic core/alumina shell nanospheres were prepared by a combination of the sol-gel process with surfactants self-assembly approach. The magnetic reduced iron core endowed the nanoparticles with magnetic properties. MFeCA possessed a uniform alumina layer of thickness $\sim 30 \mathrm{~nm}$, a large pore volume $\left(\sim 0.22 \mathrm{~cm}^{3} / \mathrm{g}\right)$ and a large surface area $\left(\sim 140 \mathrm{~m}^{2} / \mathrm{g}\right)$.

\section{Acknowledgements}

This work was supported by the Natural Science Foundation of Shandong (ZR2015BL023).

\section{References}

[1] C.Wang, L.Wang, J. Jin, et al, Probing effective photocorrosion inhibition and highly improved photocatalytic hydrogen production on monodisperse PANI@CdScore-shell nanospheres, Appli. Cata. B: Environmental. 188(2016)351-359.

[2] W. X. Zhang, J.Z.Zheng,J. G. Shi, et al. Nafion covered core-shell structured Fe3O4@graphene nanospheres modified electrode for highly selective detection of dopamine,Anal. Chimi. Acta. 853(2015)285-290.

[3] L. L. Chen, X. D. Li, Y. Q. Wang, et al, Low-temperature synthesis of tin dioxide hollow nanospheres and their potential applications in dye-sensitized solar cells and photoelectrochemical type self-powered ultraviolet photodetectors, J. Power. Sources. 272(2014)886-894.

[4] S. S.Abdelnasser,A. A. Ibrahim, et al, Cyclodextrin glucanotransferase immobilization onto functionalized magnetic double mesoporous core-shell silica nanospheres, Electron. J. Biotech. 17(2014)55-64.

[5] B. Cheng, L. Zhao, J. G. Yu , et al, Facile fabrication of $\mathrm{SiO}_{2} / \mathrm{Al}_{2} \mathrm{O} 3$ composite microspheres with a simple electrostatic attraction strategy, Mater. Res. Bull. 43(2008)714-722.

[6] W. R. Zhao, J. L. Gu,L. X. Zhang, et al, Fabrication of uniform magnetic nanocomposite spheres with a magnetic core/mesoporous silica shell structure, J. Am. Chem. Soc. 127(2005), 8916-8917.

[7] Y. H. Deng, D. W. Qi, C. H. Deng, et al, Superparamagnetic high-magnetization microspheres with a Fe3O4@SiO2 core and perpendicularly aligned mesoporous $\mathrm{SiO} 2$ shell for removal of microcystins, J. Am. Chem. Soc.130(2008)28-29. 
[8] J. T. Feng, Y. J. Lin, F. Li, et al, Preparation, structure and properties of micro-spherical alumina with magnetic spinel ferrite cores, Appl. Catal .A Gen. 329(2007)112-119.

[9] J. T. Feng, Y. J. Lin, F. Li, et al, Preparation and properties of microspherical alumina with a magnetic core/shell structure, Ind. Eng. Chem. Res. 48(2009)692-697.

[10] Y. Li, Y. C. Liu, J. Tang, et al, Fe3O4@Al2O3 magnetic core-shell microspheres for rapid and highly specific capture of phosphopeptides with mass spectrometry analysis, J. Chromatogr. A.1172( 2007)57-71.

[11] X. H.Guo, Y. H. Deng, D. Gu, et al. Synthesis and microwave absorption of uniform hematite nanoparticles and their core-shell mesoporous silica nanocomposites, J. Mater.Chem. 19(2009)6706-6712.

[12] S. J. Gregg, K. S. W. Sing, Adsorption, surface area and porosity, second ed., Academic Press, New York, 1982.

[13] V. Frederic, K .Shervin, E. D. Mark, Synthesis of pure alumina mesoporous materials, Chem. Mater. 8(1996)1451-1464.

[14]M. Ohmori, E. Matijevic, Preparation and properties of uniform coated inorganic colloidal particles: 8. silica on iron, J .Colloid. Interface. Sci.160(1993)288-292. 